See discussions, stats, and author profiles for this publication at: https://www.researchgate.net/publication/323674208

\title{
High-speed water impacts of flat plates in different ditching configuration through a Riemann-ALE SPH model
}

Article in Journal of Hydrodynamics · February 2018

Dol: 10.1007/542241-018-0004-y

\section{CITATION}

1

5 authors, including:

Salvatore Marrone

Italian National Research Council

53 PUBLICATIONS 1,323 CITATIONS

SEE PROFILE

Matthieu De Leffe

Nextflow Software

17 PUBLICATIONS 102 CITATIONS

SEE PROFILE
Andrea Colagrossi

Italian National Research Council

106 PUBLICATIONS 3,323 CITATIONS

SEE PROFILE

5. David Le Touzé

Ecole Centrale de Nantes

153 PUBLICATIONS 1,610 CITATIONS

SEE PROFILE

Some of the authors of this publication are also working on these related projects:

Project Multiphase flow using ISPH View project

Project EPFL LMH research View project 


\title{
High-speed water impacts of flat plates in different ditching configuration through a Riemann-ALE SPH model
}

\author{
S. Marrone ${ }^{1}$, A. Colagrossi ${ }^{1}$, L. Chiron ${ }^{2}$, M. De Leffe ${ }^{2}$, D. Le Touzé $e^{3}$, \\ 1. CNR-INSEAN, Marine Technology Research Institute, Rome, Italy \\ 2. NEXTFLOW Software, Nantes, France \\ 3. LHEEA Lab. (UMR CNRS), Ecole Centrale de Nantes, Nantes, France \\ E-mail: salvatore.marrone@cnr.it
}

\begin{abstract}
The violent water entry of flat plates is investigated through a Riemann-ALE SPH model. The test conditions are of interest for problems related to aircraft and helicopter emergency landing in water. Three main parameters are considered: the horizontal velocity, the approach angle (i.e. vertical to horizontal velocity ratio) and the pitch angle, $\alpha$. Regarding the latter, small angles are considered in this study. As described in the theoretical work by Zhao and Faltinsen (1993), for small $\alpha$ a very thin, high-speed jet of water is formed, and the time-spatial gradients of the pressure field are extremely high. These test conditions are very challenging for numerical solvers. In the present study an enhanced SPH model is firstly tested on a purely vertical impact with deadrise angle $\alpha=4^{\circ}$. An in-depth validation against analytical solutions and experimental results is carried out, highlighting the several critical aspects of the numerical modelling of this kind of flow, especially when pressure peaks are to be captured. A discussion on the main difficulties when comparing to model scale experiments is also provided. Then, the more realistic case of a plate with both horizontal and vertical velocity components is discussed and compared to ditching experiments recently carried out at CNR-INSEAN. In the latter case both $2 \mathrm{D}$ and 3D simulations are considered and the importance of 3D effects on the pressure peak is discussed for $\alpha=4^{\circ}$ and $\alpha=10^{\circ}$.
\end{abstract}

Key words: Aircraft ditching, High-speed water entry, Smoothed Particle Hydrodynamics

\section{Introduction}

The problem of the high-speed water entry is classically of interest in the naval field as far as slamming loads on ships are concerned. In this context several theoretical solution have been derived for simplified conditions and a large literature of experimental data is available. Water-entry problems are also very important in the aircraft ditching, that is, the emergency landing on water. The response of the vehicle to this kind of water impact is critical in terms of safety of the passengers and certifications issued by airworthiness authorities includes the success of the airframe in ditching tests. In this context few high-fidelity numerical methods have been developed so far (examples can be found in [1], [2]).

The SPH method has already shown promising results for the simulation of violent water impacts thanks to its accuracy and easiness in following the free-surface deformations (see e.g. [3]-[5]). In the present work an in-depth study and validation of the SPH model is provided for $2 \mathrm{D}$ water entries of flat panels with small

*Project supported by the European Union's Horizon 2020 research and innovation programme under grant agreement No 724139.

Biography: Salvatore Marrone (1983), Male, Ph. D.

Corresponding author: Salvatore Marrone,

E-mail: salvatore.marrone@cnr.it deadrise angle. Both purely vertical and oblique impact velocity with high horizontal velocity component are studied (sections 3 and 4). These conditions are of interest for, respectively, helicopter and airplane ditching situations. To this aim the numerical outcome will be compared to experimental measurements and analytical solutions when available. The influence of the 3D effects are also addressed for the oblique water entry through a 3D SPH model. In order to accurately resolve the high and localized pressure peaks developed at the impact a Riemannbased SPH solver is used within an Arbitrary Eulerian- Lagrangian (ALE) framework. The choice of the numerical parameters to be adopted, as e.g. the liquid compressibility, is critically and extensively discussed on the base of physical considerations peculiar of water-impact flows.

\section{Adopted SPH scheme}

In the present work Euler equations for compressible fluids are solved. Indeed, since the Reynolds number of the flow is quite high and only the impact stage is simulated (short time- range regime) viscous effects can be considered negligible. The weaklycompressible model is adopted; the fluid is, therefore, assumed to be barotropic and a classical stiffened state equation is used: 


$$
p(\rho)=\frac{c_{0}^{2} \rho_{0}}{\gamma}\left[\left(\frac{\rho}{\rho_{0}}\right)^{\gamma}-1\right]+p_{0}
$$

where $\rho_{0}$ and $\mathrm{p}_{0}$ are constant, $\mathrm{c}_{0}$ is the speed of sound, and $\gamma$ is a dimensionless parameter greater than 1 (in all of the following examples $\gamma=7$ is used).

When considering violent free-surface flows, the proper identification of the reference velocity $\mathrm{U}_{\text {ref }}$ is crucial, as discussed in the following sections. Considering the Mach number $\mathrm{Ma}=\mathrm{U}_{\text {ref }} / \mathrm{c}_{0}$, the constraint $\mathrm{Ma}<0.1$ is is enforced to make compressibility effects negligible. Additionally, during violent impact events (i.e., flat impacts) the acoustic pressure $\mathrm{p}=\rho \mathrm{uc}_{0}$ can be reached, and in this case the pressure peak intensity becomes proportional to $1 / \mathrm{Ma}$. On the other hand, in such a condition an incompressible constraint can induce singularities on the pressure field (see [6] for a discussion on the difference between these two models in impact situations). This is linked to the fact that for this kind of impacts the presence of the air phase is generally crucial and the single- phase approach can lead to incorrect pressure evaluations under the incompressible/weakly-compressible hypothesis (for a deeper discussion see also [4]). Being aware of these limits of the single-phase model, the results obtained in this paper have been produced considering a possible Mach dependency.

In the present work the Riemann-based solver described in [7] is adopted. In that work the ALE formalism is used allowing for maintaining a regular particle spatial distribution and smooth pressure fields while preserving the whole scheme conservation and consistency of the classical SPH scheme. The introduction of the Riemann-based solver in the SPH scheme leads to an increased stability and robustness of the scheme with respect to the standard SPH formulation. The formalism proposed by [8], Thanks to the introduction of Riemann-solvers the fluxes between particles are upwind oriented and the resulting scheme is characterized by good stability properties. The discrete Euler equations are written as follows:

$$
\left\{\begin{array}{c}
\frac{D \boldsymbol{r}_{i}}{D t}=\boldsymbol{v}_{0 i} \\
\frac{D V_{i}}{D t}=V_{i} \sum_{j}\left(\boldsymbol{v}_{0 j}-\boldsymbol{v}_{0 i}\right) \boldsymbol{\nabla} W_{i j} V_{j} \\
\frac{D\left(V_{i} \rho_{i}\right)}{D t}=-V_{i} \sum_{j} 2 \rho_{E}\left(\boldsymbol{v}_{\boldsymbol{E}}-\boldsymbol{v}_{0}\left(\boldsymbol{r}_{i j}\right)\right) \boldsymbol{\nabla} W_{i j} V_{j} \\
\frac{D\left(V_{i} \rho_{i} \boldsymbol{v}_{i}\right)}{D t}=-V_{i} \sum_{j} 2\left[\rho_{E} \boldsymbol{v}_{\boldsymbol{E}} \otimes\left(\boldsymbol{v}_{\boldsymbol{E}}-\boldsymbol{v}_{0}\left(\boldsymbol{r}_{i j}\right)\right)\right] \boldsymbol{\nabla} W_{i j} V_{j} \\
-V_{i} \sum_{j} 2 P_{E} \boldsymbol{I} \boldsymbol{\nabla} W_{i j} V_{j}+\omega_{i} \rho_{i} \boldsymbol{g}
\end{array}\right.
$$

where $\rho_{E}, P_{E}$ and $v_{E}$ are the solutions of the Riemann problem at the interface $\mathbf{r}_{\mathrm{ij}}=\left(\mathbf{r}_{\mathrm{i}}+\mathbf{r}_{\mathrm{j}}\right) / 2$, between particles $i$ and $j$. The particle transport velocity $\mathbf{v}_{0}$ is obtained as the summation of the particle velocity plus a small perturbation which helps preserving a regular particle distribution (details about the adopted model can be found in [7]).

\section{Vertical water entry of a flat panel}

In this first section the vertical impact of a flat plate is numerically investigated and validated. Specifically, results of the 2D single-phase simulations are described and compared to the experimental data from a wet drop test performed in [9]. In that work a flat panel (panel length $\mathrm{L}$ equal to $64 \mathrm{~cm}$ ) impacting with a deadrise angle of $4^{\circ}$ and a vertical impact velocity $U$ of $6.0 \mathrm{~m} / \mathrm{s}$ is studied. Measures of pressures at several positions along the plate are taken, allowing for a detailed control of the pressure peak repeatability and for possible 3D effects.

As described in the theoretical work by [10], for these small deadrise angles a very thin, high-speed jet of water is formed, and the time-spatial gradients of the pressure field are extremely high. This makes the test conditions very demanding for numerical solvers. From the potential flow theory by [10], the jet thickness at model scale is about $0.1 \mathrm{~mm}$. It is worth noting that the theory in [10] is formulated for symmetric wedge impacts whereas in the present case the impact of a inclined single plate is considered. More details about the reference solution to be adopted are given in section 3.2.

On the base of this theoretical data, the 2D simulation has been conducted using a very high spatial resolution, corresponding to a particle size $\Delta \mathrm{x}=$ $15.6 \mu \mathrm{m}$; by referring the latter to the panel length $\mathrm{L}$, the ratio $\mathrm{L} / \Delta \mathrm{x}$ is equal to 41,000 . The whole tank depth and width are, respectively, $3 \mathrm{~m}$ and $6 \mathrm{~m}$. In order to manage such a small particle size a variable-h technique has been used. Specifically, the particle size gradually changes with a maximum magnification factor of 3,200 between the most refined region and the lowest resolution one (see figure 1). The total number of particles is about 3 million.

For small dead-rise angles water compressibility cannot be neglected when calculating the speed of the water jet $U_{\text {jet }}$ (see e.g. [11]-[13]). In the experimental conditions the speed of sound in water is $\mathrm{c}_{0}^{*}=$ $1481 \mathrm{~m} / \mathrm{s} \simeq 247 \mathrm{U}$, therefore the Mach number $\mathrm{Ma}=\mathrm{U} / \mathrm{c}_{0}^{*}$ is about $4 \times 10^{-3}$. Under such a condition an estimate of the jet speed is Ujet $42 \mathrm{U} \sim 250 \mathrm{~m} / \mathrm{s}$.

As noted in [11], this very thin, high-speed jet disintegrates at some distance from the root due to interaction with both the surrounding air and the 
surface of the body. In this case surface tension plays an important role on the jet evolution. This local and complex physics is not taken into account in the present numerical method but it is not supposed to play a relevant role on the pressure distribution.

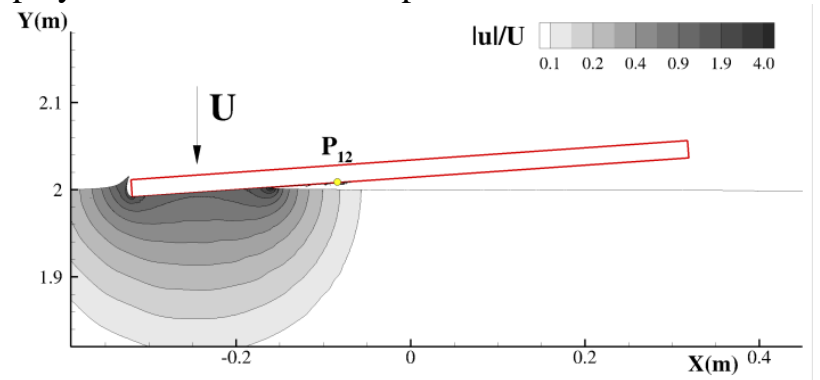

Fig. 1: Vertical impact of a flat panel. Contour lines are representative of the SPH velocity intensity. Pressure probe number 12, which is the gauge used for the comparison with the experimental data, is also depicted.

\subsection{Selection of the speed of sound for the SPH model}

Before starting the SPH simulations the speed of sound needs to be specified. As mentioned in section 2 , the numerical Mach number usually adopted within the $\mathrm{SPH}$ simulations is $\mathrm{Ma}=\mathrm{U} / \mathrm{c}_{0}=0.1$, which guarantees the weakly-compressible regime (i.e., compressibility plays a negligible role). Nonetheless, for this kind of impact the reference speed for the Mach number cannot be the impact velocity U. Indeed, considering that the intersection point between the horizontal undisturbed free-surface and the wedge surface has a speed equal to $\mathrm{U}_{\text {inters }}=\frac{\mathrm{U}}{\sin (\alpha)} \simeq 14 \mathrm{U}$. According to Wagner theory the water jet formed during the impact has a speed higher than $U_{\text {inters }}$. Therefore, if one chooses the speed of sound using the wedge speed $\mathrm{U}$, i.e., $\mathrm{c}_{0}=10 \mathrm{U}$, the jet would not form at all, its speed being in the supersonic regime. This is a clear example where the weakly-compressible rule $\mathrm{Ma} \leqslant 0.1$ needs to be enforced in a proper way, considering the specific problem at hand.

In the present case the reference speed should be the water jet speed $U_{\text {jet }}$ which, however, is an unknown of the problem. Using the theory in [11], the estimate $U_{\text {jet }}$ $=40 \mathrm{U}$ can be used. Note that, using the latter constraint, the speed of sound in water would result even higher than the real one, $\mathrm{c}_{0}=400 \mathrm{U}$ versus $\mathrm{c}_{0}=$ 247U. This means that for the adopted model scale water compressibility effects are not negligible, at least inside the jet region. However, considering that in the jet zone the pressure is close to the ambient pressure, water compressibility effects should not play a relevant role on the local impact loads.

In order to satisfy the weakly-compressible assumption, at least in the impact region, the reference velocity used is $U_{\text {ref }}=P_{\max } / \rho$, where $\mathrm{P}_{\max }$ is an unknown of the problem and needs to be estimated. Then, an ex-post facto verification of the SPH simulations is required. Using the Wagner theory (which is valid for small deadrise angles as far as the air presence is negligible) the maximum pressure predicted for $\alpha=4^{\circ}$ is:

$$
P_{\text {max }}=\frac{1}{2} \rho U^{2} \frac{\pi^{2} / 4}{[\tan (\alpha)]^{2}} \simeq 252 \rho U^{2}
$$

corresponding to about 91 bar in our experiment.

The water-hammer pressure for this impact is $\rho c_{0}^{*} \mathrm{U}=88$ bar which is the maximum pressure level that can be physically reached in the experiments. The $\mathrm{P}_{\max }$ predicted by potential flow theory is higher than the acoustic pressure, and this is a further indication that water compressibility cannot be neglected for this problem. Thus, considering $\mathrm{P}_{\max } \sim 80$ bar, the reference velocity is $\mathrm{U}_{\text {ref }}=\mathrm{P}_{\max } / \rho \sim 15 \mathrm{U}$ which is smaller than $\mathrm{U}_{\text {jet }}=40 \mathrm{U}$.

Taking this into account, a good compromise for the SPH speed of sound can be obtained by adopting a reference velocity $U_{\text {ref }}=10 \mathrm{U}$ which implies a speed of sound $\mathrm{c}_{0}=100 \mathrm{U}$ (i.e., Mach number $\mathrm{Ma}=$ $\left.\mathrm{U} / \mathrm{c}_{0}=0.01\right)$. The resulting time step is equal to $\Delta \mathrm{t}=1.5 \Delta \mathrm{x} / \mathrm{c}_{0} \simeq 0.04 \mu \mathrm{s}$ which is 250 times smaller than the sampling rate of the pressure probes used in the experiments (i.e., the SPH sample frequency is $25 \mathrm{MHz}$ versus the $100 \mathrm{kHz}$ of the experimental pressure probes). This aspect is expected to influence the observed pressure peak which, for the considered configuration, needs a high time/space resolution to be captured. Further, in order to verify the appropriateness of such a choice, also a simulation using $\mathrm{c}_{0}=247 \mathrm{U}$ has been run, the results will be shown at the end of section 3.2.

\subsection{Comparison between SPH results, analytical solution and experimental data}

Figure 2 shows the pressure field predicted by the SPH for the flat panel impact. In the same plot the free surface deformation evaluated by the potential flow theory by [10] is reported. Left plot of figure 3 shows an enlarged view of the flow velocity predicted by the

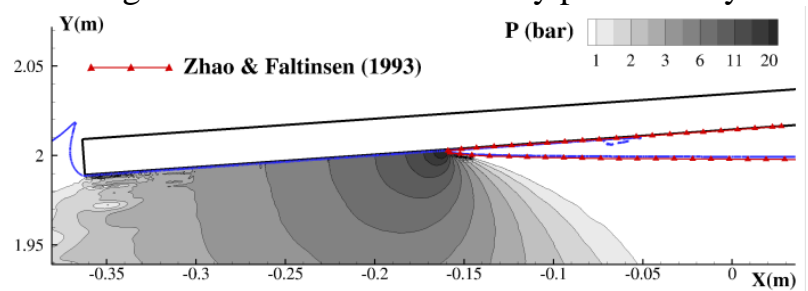

Fig. 2: Vertical impact of a flat panel: contour lines are representative of the SPH pressure field. Red solid line is the free surface extracted from [10]. 


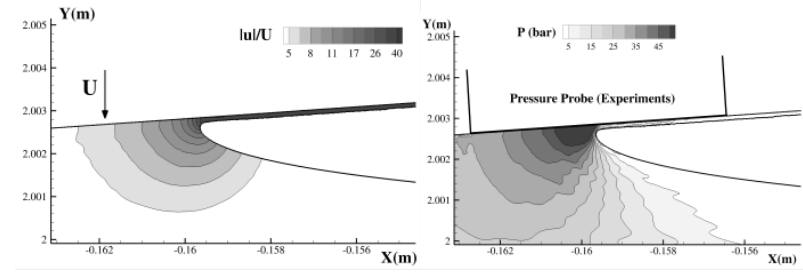

Fig. 3: Vertical impact of a flat panel: enlarged view of the impact zone. Left: contours of the velocity module. Right: contours of the SPH pressure field. The size of the pressure gauges used in the experiments is also depicted.

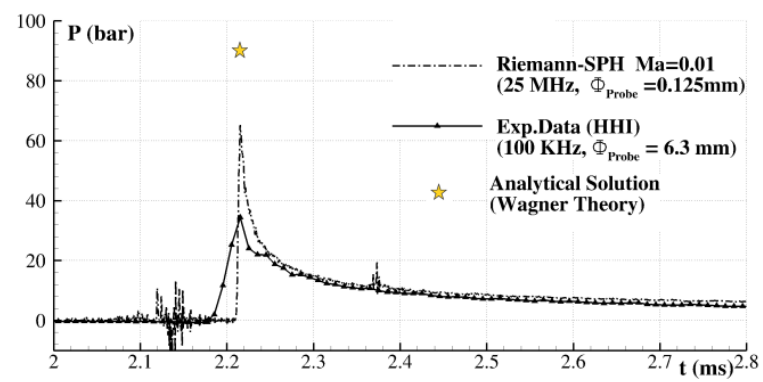

Fig. 4: Vertical impact of a flat panel: SPH pressure time histories at probe $\mathrm{P} 12$ compared with experimental data and with analytical solution Wagner theory.

SPH in the area of the highest pressure levels. A thin water jet is formed with a thickness of about $0.1 \mathrm{~mm}$ corresponding to about ten particles, thus justifying the high $L / \Delta x$ ratio needed to properly solve such a flow.

In the right plot of the same figure an enlarged view of the pressure field is shown (in this case the displayed pressure range is enlarged too). From this plot it is seen that the high-pressure region is limited to an area of $1 \mathrm{~mm}^{2}$ with a pressure peak of about 60 bar. In the same figure the size of the probe used in the experiments is depicted. Clearly, the pressure sensor has a size much larger than the pressure bulb formed below the water-jet root. This aspect will be discussed in the following section.

In figure 4 the time histories of the pressure measured at probe $\mathrm{P} 12$ are shown. This probe is positioned 23.6 $\mathrm{cm}$ from the left-hand edge of the panel (see figure 1). The SPH solution is compared with the experiments and with the pressure peak predicted by Wagner theory. The SPH prediction is between the two reference data sets, and is characterized by high-frequency components due to the fragmented jet of water that pass over the numerical pressure probe. The latter has a dimension of $0.125 \mathrm{~mm}$ (which is the size of the SPH kernel support) and the SPH sampling rate is $25 \mathrm{MHz}$. Both the SPH and the analytical predictions overestimate the experimental data for which the maximum pressure recorded is 34 bar (in the SPH solution the pressure peak reaches 70 bar whereas the analytical prediction is 91 bar). It has to be noted that, even though the Wagner theory is referred to the case of a symmetric wedge entry, according to [14] and [15] the value of the pressure peak should be substantially the same of the case of an oblique flat panel impact (it will be shown in section 3.3 that this approximation can be quite rough for the present case).

Conversely, regarding the entire pressure signal, it is not possible to compare to classical analytical solutions, such as in [10], since they are all formulated for the symmetric wedge entry case.

As mentioned above, air entrainment is expected to play a minor role for deadrise angles greater than $3^{\circ}$ [10], [15]. Notwithstanding that, most of the experimental measurements available in the literature for angles close to $4^{\circ}$ exhibit pressure peaks much smaller than the one predicted by potential theory (cf., [15]-[18]) and the values measured in the present study are in fair agreement with previous experiments by [15], [16]. As for possible 3D effects, these have been checked by comparing the pressure values on gauges aligned at the same distance from the piercing edge. For the probes positioned in the most central region no relevant differences have been observed. However, the maximum pressure impact measured in the experiment for small dead-rise angles can be also affected by:

1) Changes of the body velocity during the impact stage;

2) Rotations of the body during the impact stage;

3) Deformations of the wedge surface;

4) Sampling rate of the pressure signals;

5) Size of the pressure gauge;

Thanks to the experimental setup adopted the first three points can be neglected, while the last two can play an important role. In order to take into account the experimental sampling rate, the SPH signal has first been filtered using a moving average filter (MAF) reducing the numerical sampling rate from $25 \mathrm{MHz}$ to $100 \mathrm{kHz}$. The result is illustrated in figure 5. The reduction of the SPH peak due to this filtering procedure is not enough to get a good agreement with the experimental data. As a further step the SPH pressure has been measured integrating on a circular area equal to the size of the experimental pressure probes and then filtered at $100 \mathrm{kHz}$. This result is

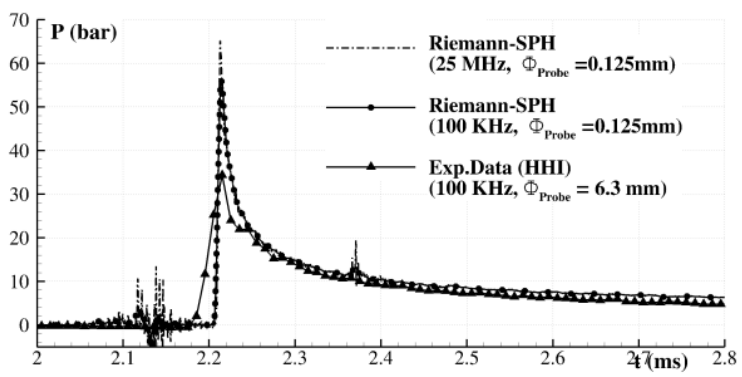

Fig. 5: Vertical impact of a flat panel: SPH pressure time histories versus experimental data. The SPH signal is filtered 
with a running average filters (MAF) at $100 \mathrm{kHz}$ (dashed-dotted line).

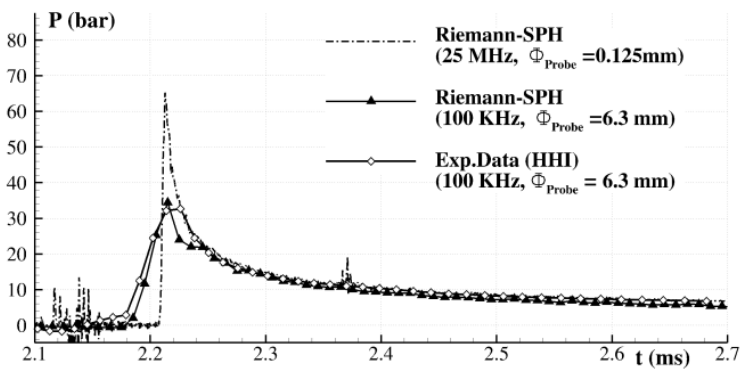

Fig. 6: Vertical impact of a flat panel: SPH pressure time histories versus experimental data. The SPH signal recorded using the size of the experimental pressure gauges and filtered at $100 \mathrm{kHz}$ is also shown.

shown in figure 6 . In this case the SPH output is much closer to the pressure recorded in the experiment.

Summarizing, according to the analytical solution very narrow pressure peaks are expected for this kind of impact. Using pressure gauges with a size of few millimeters and a sampling rate of $100 \mathrm{kHz}$ it is not possible to record such a localized event. Having in mind such limits, through SPH it is possible to get predictions close to the experimental data if the pressures are integrated over the experimental gauge area, even when using a speed of sound $c_{0}$ smaller than the real one $c_{0}^{*}$.

In figure 7 the experimental pressure time histories recorded on a sequence of pressure probes is reported. Even if the experimental pressure peaks present some fluctuations, very similar pressure evolutions are recorded with an almost constant time shift at each probe. The SPH results show quite good repeatability of the pressure peaks along the wedge surface as well (see figure 8). In this regard, it is worth comparing the propagation velocity of the pressure peak along the plate, $U_{P}$. Indeed, in water entry flows the value of the pressure peak should correlate to $\mathrm{U}_{\mathrm{P}}^{2}$ (see e.g. [19], [20]) as:

$$
C_{P}=\frac{P_{\max }}{\frac{1}{2} \rho U_{P}^{2}}=1
$$

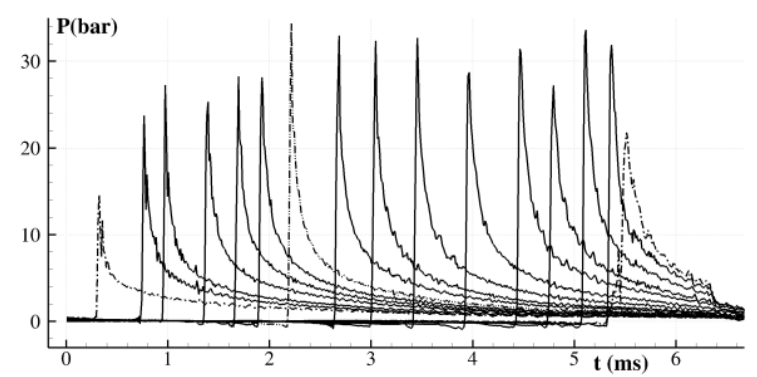

Fig. 7: Vertical impact of a flat panel: experimental data for the pressure time histories on a row of probes (not equispaced). The time history from the first and last probes on the panel impacting the water are colored in red. The time history in blue is from probe $\mathrm{P} 12$, used in the previous figures for comparison with the SPH solution.

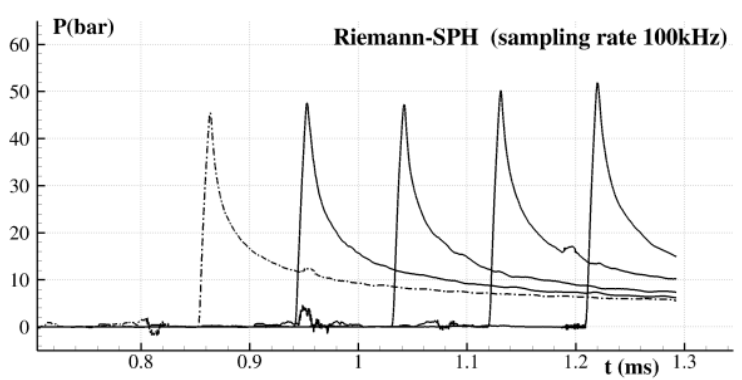

Fig. 8: Vertical impact of a flat panel: SPH pressure time histories on a row of probes. The original SPH signals have been filtered with a MAF at $100 \mathrm{kHz}$ as in the experimental signals. The time history from the first probe on the panel impacting the water is plotted with the dashed line.

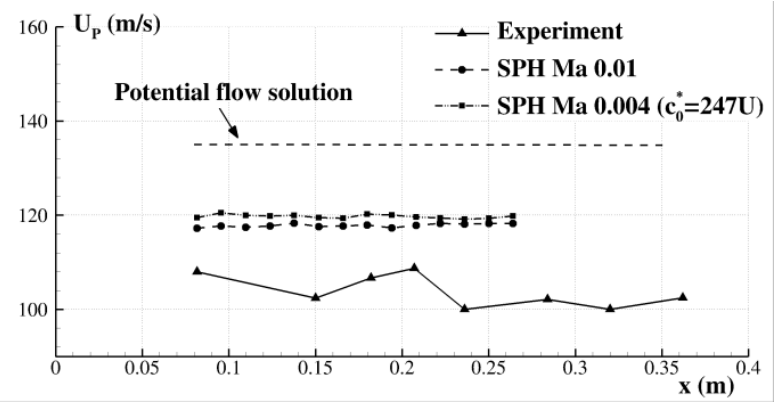

Fig. 9: Vertical impact of a flat panel: Peak propagation velocity $U_{P}$ calculated on several positions along the panel for the experiment (triangles), SPH simulation at $\mathrm{Ma}=0.01$ (circles) and $\mathrm{Ma}=0.004$ (squares), and analytical value from potential flow theory (dashed line). The origin of the $\mathrm{x}$-axis is set at the left-hand edge of the panel.

\begin{tabular}{lcccc} 
& $U_{P}(\mathrm{~m} / \mathrm{s})$ & $1 / 2 \rho U_{P}^{2}($ bar $)$ & $P_{\max }($ bar $)$ & $C_{P}$ \\
\hline Exp. & 104 & 54 & 30 & 0.56 \\
SPH Ma $=0.01$ & 117 & 68 & 70 & 1.03 \\
SPH Ma $=0.004$ & 119 & 71 & 75 & 1.06 \\
\hline
\end{tabular}

Table 1: Average of the peak propagation velocity $U_{P}$ plotted in fig. 9. The corresponding pressure peak $1 / 2 \rho \mathrm{U}_{\mathrm{P}}^{2}$, the actual average pressure peak measured at the probes $\mathrm{P}_{\max }$ and the related pressure coefficient $C_{P}(4)$ are also reported.

In figure 9 the calculated values of $\mathrm{U}_{\mathrm{P}}$ for both $\mathrm{SPH}$ and experimental results are reported for several probes. Only the probes far from the panel edges have been considered to avoid influences of either the initial impact stage or the final stage, for which the flow self-similarity is not applicable. In the same figure the values of $U_{P}$ obtained from a simulation adopting the real speed of sound $c_{0}^{*}=247 \mathrm{U}(\mathrm{Ma}=0.004)$ are also reported.

The difference between the simulation with $\mathrm{Ma}=0.01$ and $\mathrm{Ma}=0.004$ is very small (about $2 \%$ of the average value), confirming that within the weakly- compressi- 
ble regime the Mach number effect is limited. For the sake of completeness

in the same plot also the analytical value of $U_{P}$ (valid for a wedge entry problem) is reported, i.e.:

$$
U_{P}=\frac{\pi}{2} U \cot \alpha
$$

In Table I The average values of $U_{P}$ are reported. For the simulation at $\mathrm{Ma}=0.01$ the average value of $U_{P}$ is about $117 \mathrm{~m} / \mathrm{s}$ corresponding to a pressure coefficient $\mathrm{C}_{\mathrm{P}}=1.03$ which is close to the expected value (eq. 4). On the other hand, since the value of $U_{P}$ predicted by the SPH is smaller than the analytical one, maximum pressure of the SPH cannot be equal to the one predicted by the Wagner theory as shown above. This difference is essentially due to the fact that Wagner theory is referred to a symmetric wedge entry, while the present case is asymmetric (see section 3.3).

When considering the experimental pressure probes an average value of $U_{P}$ equal to $104 \mathrm{~m} / \mathrm{s}$ is obtained (with a standard deviation $\sigma=3.1$ ). This propagation velocity of the pressure peak corresponds to a pressure coefficient $C_{P}$ equal to 0.56 . This confirms that the measurement system adopted is not able to record the real pressure peaks which are therefore underestimated as shown in this section.

\subsection{Comparison between symmetric and asymmetric water entry}

In the last subsection it has been shown that the pressure peak predicted by SPH is consistent with the water impact theory. Indeed, the calculated peak propagation velocity $\mathrm{U}_{\mathrm{P}}$ and the pressure peak $\mathrm{P}$ max give a pressure coefficient $C_{P}$ close to unity (see eq. 4 ). Nonetheless, it still remains a significant discrepancy between SPH results and the analytical solution for this impact angle. In order to investigate the source of this incongruity a further simulation has been performed. The symmetric problem has been set by retaining the initial particle configuration of the asymmetric case and closing the fluid domain at the left edge of the panel with a vertical wall (see figure 10). The simulation has been run with $\mathrm{Ma}=0.01$.

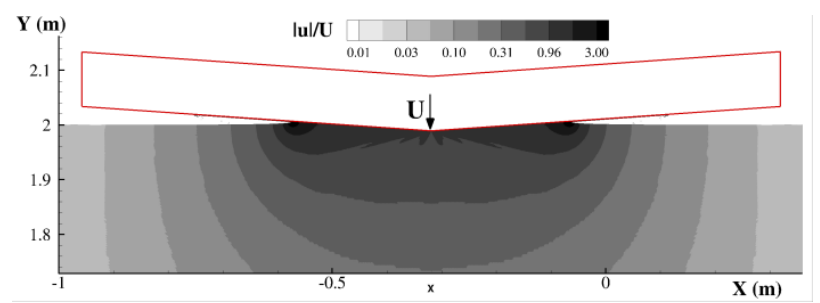

Fig. 10: Vertical impact of a flat panel: fluid domain adopted in the symmetric problem configuration. Contours refer to the module of the velocity field.

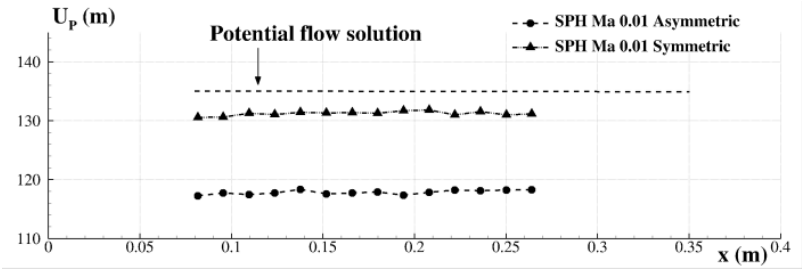

Fig. 11: Vertical impact of a flat panel: Peak propagation velocity $U_{P}$ calculated on several positions along the panel for the asymmetric (circles) and symmetric (triangles) SPH simulations, and analytical value from potential flow theory (dashed line). The origin of the $\mathrm{x}$-axis is set at the left-hand edge of the panel.

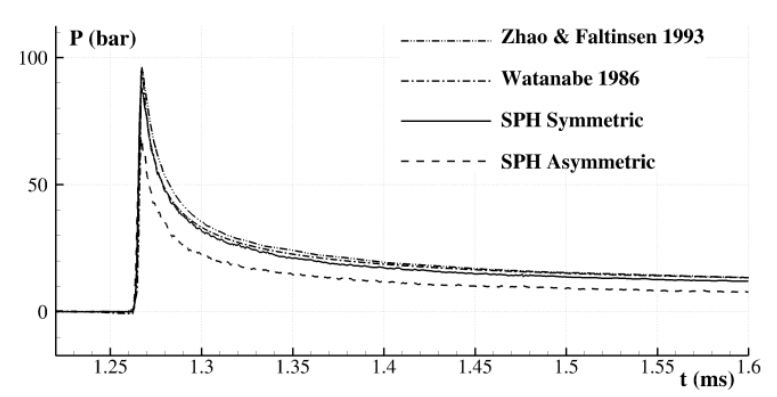

Fig. 12: Vertical impact of a flat panel: pressure time histories of symmetric (solid line) and asymmetric (dashed) solutions measured at $0.16 \mathrm{~m}$ from the left-hand edge of the panel. Analytical solutions by [21] (dash-dot line) and by [10] (dash dot-dot line) are also reported.

In figure 11 the peak propagation velocity for both symmetric and asymmetric problems are shown. It is clear that in the symmetric configuration the SPH solution is now much closer to the potential flow prediction and, because of this increase in the value of $\mathrm{U}_{\mathrm{P}}$, a larger pressure peak is expected on the panel surface. The recorded pressure at $0.16 \mathrm{~m}$ from the left-hand edge of the panel is shown in figure 12 for both symmetric and asymmetric SPH solutions.

Consistently with the observed value of $\mathrm{U}_{\mathrm{P}}$, the pressure peak of the symmetric solution is about 88 bar. In the same figures the analytical solutions from [21] and [10] are also reported. Evidently, the symmetric SPH solution is now much closer to the analytical prediction and the remaining difference can be mainly attributed to fluid compressibility. This is in agreement with the analytical work in [22].

In that work it is shown that, generally, asymmetric impacts induce smaller pressure peaks with respect to the symmetric case. This effect is emphasized when the deadrise angle is smaller (in [22] the smallest angle considered is $10^{\circ}$ ).

\section{Water entry with high horizontal speed}

In this section the ditching problem including a large horizontal velocity component is studied, comparing the SPH outcome to model test experiments. Given 
the higher complexity of the problem, in this section also possible 3D effects are investigated, as their role is expected to be not negligible for this case.

\subsection{Description of the experimental data}

Guided ditching impact experiments [19], [23] were performed in the CNR-INSEAN towing tank, which is $470 \mathrm{~m}$ long, $13.5 \mathrm{~m}$ wide and $6.5 \mathrm{~m}$ deep. The dimension of the flat plate were $500 \mathrm{~mm}$ by $1000 \mathrm{~mm}$ (fig. 13). Pressures at 18 points are measured through Kulite XTL123B pressure transducers. The sampling rate of the latter is $200 \mathrm{kHz}$ while their dimension is $3.8 \mathrm{~mm}$. The horizontal and vertical velocities at the impact are respectively $40 \mathrm{~m} / \mathrm{s}$ and $-1.5 \mathrm{~m} / \mathrm{s}$. In the experiments, these velocities are assumed to remain constant during the whole ditching impact.

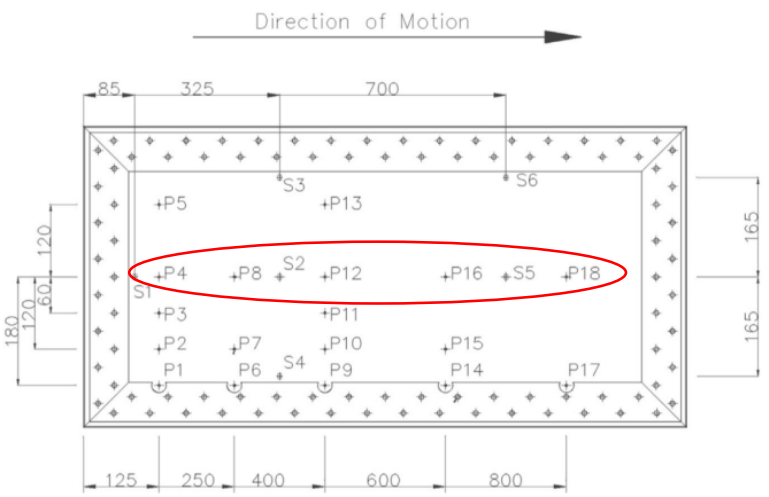

Fig. 13: Top view of the plate adopted in the ditching experiments. The ellipse shows pressure probes considered for 2D SPH simulations (measures are in $\mathrm{mm}$ ).

\subsection{Numerical methodology}

In this second test case series, simulations have been conducted using Adaptive Particle Refinement (APR) technique [24]. This technique allows keeping a high spatial resolution around the flat plate during the simulation as the refinement areas move at the same speed of the plate. In order to avoid the pressure filtering described in section 3 , the numerical adopted pressure sensor size is the same as in the experiments (i.e $3.8 \mathrm{~mm}$ ).

Given the high speeds involved in the considered ditching experiments, the simulations were performed with a nominal sound speed $\mathrm{c}_{0}{ }^{\text {water }}=1480 \mathrm{~m} / \mathrm{s}$. Comparisons with experiments are established in terms of pressure coefficient, which is defined as :

$$
C_{P}=\frac{P}{\frac{1}{2} \rho U^{2}\left[1+\left(\frac{V}{U}\right)^{2}\right]}
$$

where $\mathrm{U}$ and $\mathrm{V}$ refer respectively to the horizontal and vertical velocities.

\subsection{Impact at $4^{\circ}$ pitch angle}

The numerical tank is $20 \mathrm{~m}$ long and $6 \mathrm{~m}$ deep.
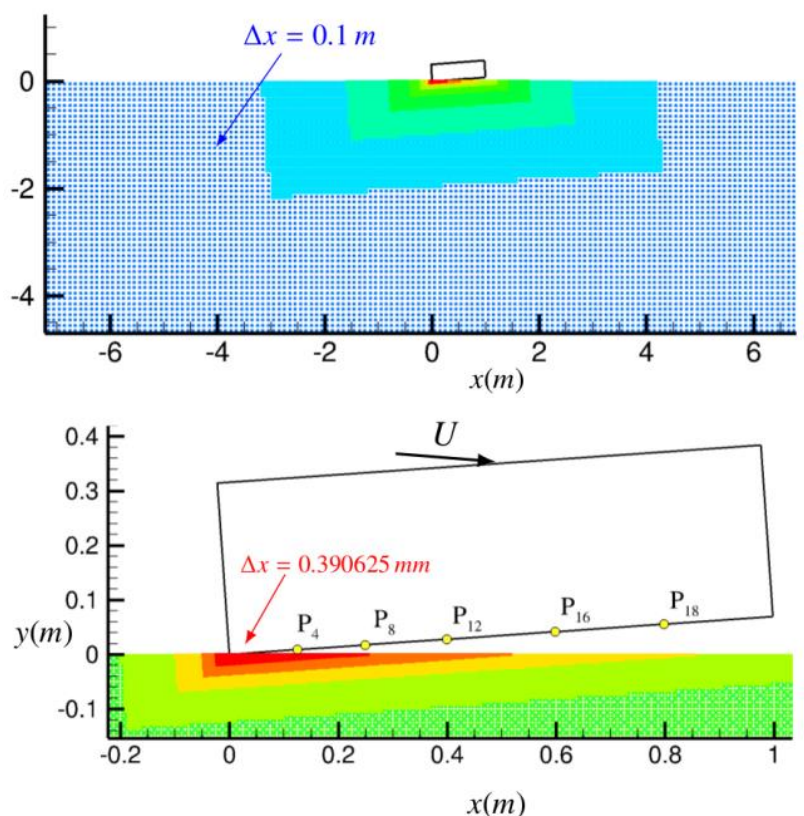

Fig. 14: Sketch of the oblique ditching simulation involving 8 refinement levels (top: global view, bottom: zoomed view). Contours refer to refinement depth.

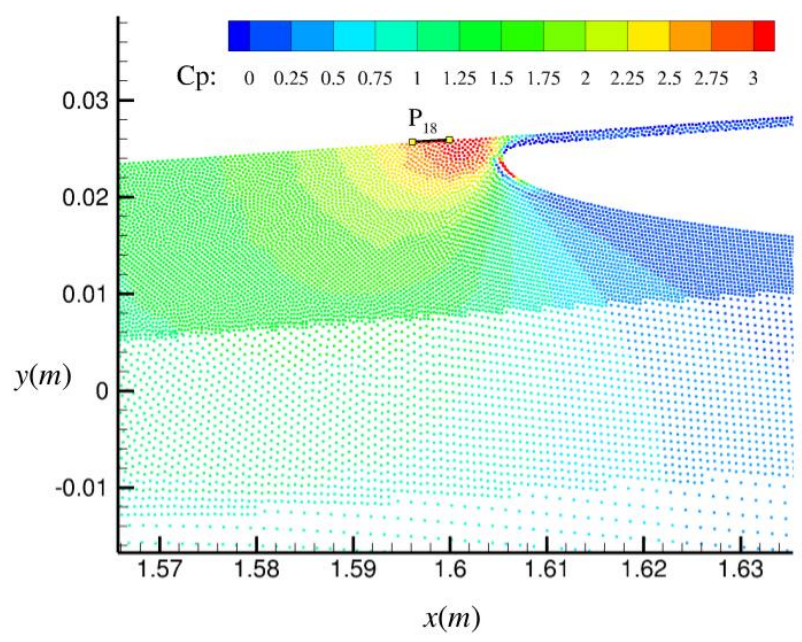

Fig. 15: Pressure coefficient field of the oblique ditching SPH simulation involving 8 refinement levels at time $\mathrm{t}=0.02 \mathrm{~s}$. The size of the pressure gauge P18 is depicted.

Simulations involving 6 refinement levels $\left(\Delta \mathrm{x}_{\min }=\right.$ $1.5625 \mathrm{~mm})$ and 8 refinement levels $\left(\Delta \mathrm{x}_{\min }=\right.$ $0.390625 \mathrm{~mm}$ ) were performed (fig. 14). At the beginning of the simulation, the total number of particles are about 67,000 and 110,000 for the test cases involving, respectively, 6 and 8 refinement levels. Figure 15 shows the pressure coefficient field predicted by the 2D SPH simulation in the area of the highest pressure levels. It is worth noting that the field appears very regular despite the contour lines cross several refinement interfaces. In the same figure also the dimension of the pressure probe is reported. 

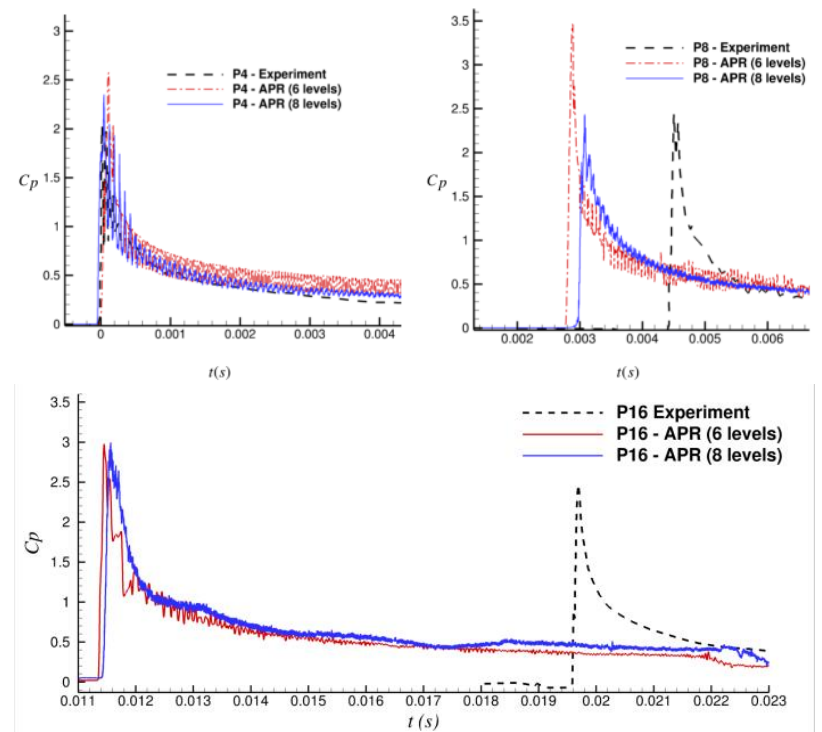

Fig. 16: Comparison between experimental data and 2D SPH simulations with different refinement levels at probes P4, P8 (top) and P16 (bottom).

In figure 16, SPH pressure time histories for three different probes are compared with the experimental data from [23]. The origin of the time axis is based on the pressure peak registered at probe P4. It can be observed that a remarkable time shift is present between the SPH and the experimental data, which increase as the plate penetrates in the water. This is clearly visible in the bottom plot of figure 16 . The time shift is due to the different peak propagation velocities between SPH and experiments. The measured peak propagation velocity in the SPH simulation is $\mathrm{U}_{\mathrm{P}}^{\mathrm{SPH}}=41.7 \mathrm{~m} / \mathrm{s}$ which is close to the value obtained from the $2 \mathrm{D}$ potential flow solution in [19] for the same case, which corresponds to $\mathrm{U}_{\mathrm{P}}^{\mathrm{POT}}=47.3 \mathrm{~m} / \mathrm{s}$. Conversely, as described in [19] the experimental data exhibit a much lower value of the propagation velocity which is mainly attributed to $3 \mathrm{D}$ effects. This aspect will be investigated more in details in the next section.

Another important aspect to discuss is the amplitude of the pressure peak. Indeed, looking at top plots of figure 16, one could be induced to conclude that the 2D SPH matches very well the 3D experimental measurements. This would be a misleading judgement since it would be in contrast with the different peak propagation velocities (according to correlation provided in equation (4) ). This apparent contradiction can be explained observing that at probe P16 (bottom plot of figure 16) the SPH pressure peak is about 50\% higher than at probes $\mathrm{P} 4$ and P8. The latter presents also more noisy signals and a less clear convergence. The reason is linked to the fact that at the beginning of the impact there are only few particles to resolve the impact region, while going forward in time the peak region grows in amplitude, i.e. it is better resolved.
Therefore, at probes $\mathrm{P} 4$ and $\mathrm{P} 8$ the SPH pressure peak is close to the experimental one because it is under resolved. Note also that the pressure peak predicted by the 2D potential flow solution in [19] is $\mathrm{C}_{\mathrm{P}}{ }^{\mathrm{max}}=4.77$. But, even at probe P16, the SPH pressure peak does not reach this value. This is due to the averaging on the experimental probe size which, similarly to vertical impact case in figure 6, largely smooth the peak. This is confirmed also by the good agreement at P16 between the solution with 6 and 8 refinement levels.

\subsection{Impact at $10^{\circ}$ pitch angle: $2 \mathrm{D}$ and $3 \mathrm{D}$ solutions}

Simulations were performed also for pitch angle of $10^{\circ}$ to investigate also possible 3D effects. Indeed, the case at $4^{\circ}$ requires a too high resolution to be studied also in 3D. The study of 3D effects is, therefore, limited to the case at $10^{\circ}$ which requires a lower computational effort. The 2D simulation has been set for this case with 6 refinement levels $\left(\Delta \mathrm{x}_{\min }=1.5625\right.$ $\mathrm{mm})$.

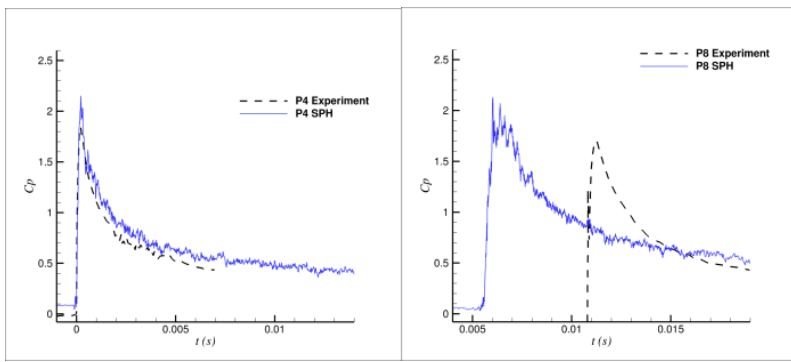

Fig. 17: Comparison between experimental data and 2D SPH simulations at probes $\mathrm{P} 4$ and $\mathrm{P} 8$ for $10^{\circ}$ pitch angle.

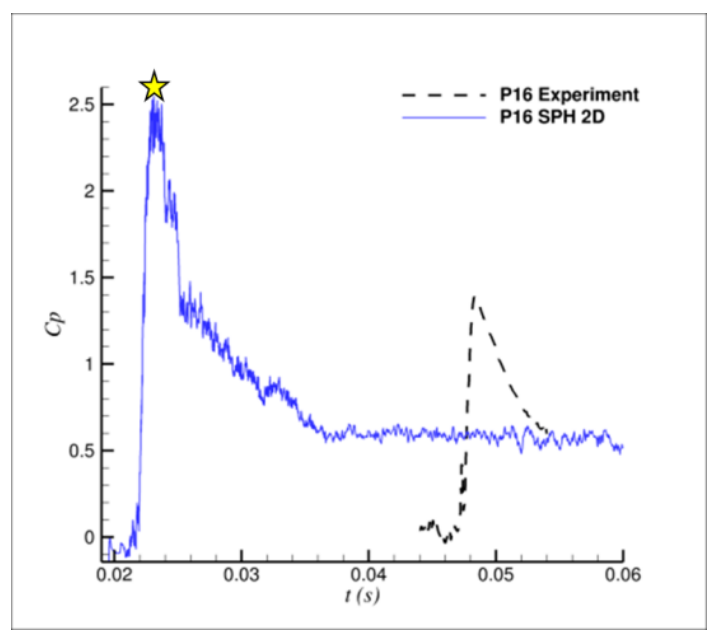

Fig. 18: Comparison between experimental data and 2D SPH simulations at probes $\mathrm{P} 16$ for $10^{\circ}$ pitch angle. The symbol refers $t$ to the pressure peak predicted by the potential flow solver in [19].

In figure 17 the 2D SPH pressure signals registered at probes $\mathrm{P} 4$ and $\mathrm{P} 8$ are reported. The behavior is similar to the case at $4^{\circ}$ : the pressure peak is close to the 
experimental one but the peak propagation velocity is different. At probe P16 (figure 18) the SPH pressure peak is, again, much higher with respect to probes $\mathrm{P} 4$ and $\mathrm{P} 8$ and is quite far from experimental data. However, the matching with the potential solution provided in [19] (represented by a star in figure 18) in this case is very good. Therefore, at $10^{\circ}$ pitch angle the averaging over the probe area seems to not affect the agreement between the SPH and the potential flow solution. The reason for that is to be found in the shape of the pressure peak, which at $10^{\circ}$ is less impulsive and much smoother than that at $4^{\circ}$.

As for the peak propagation velocity, the potential solution predicts $\mathrm{U}_{\mathrm{P}}^{\mathrm{POT}}=23.6 \mathrm{~m} / \mathrm{s}$ which is in good agreement with the $\mathrm{SPH}$ value $\mathrm{U}_{\mathrm{P}}^{\mathrm{SPH}}=23.9 \mathrm{~m} / \mathrm{s}$. Therefore, it is confirmed that, as for the oblique impact at $4^{\circ}$ in section 4.3 , for the $2 \mathrm{D}$ simulation the SPH agrees with the potential solution and remains far from the experimental data.

The 3D simulation of the case at $10^{\circ}$ pitch angle has been run with 7 refinement levels, the minimum particle spacing being $\Delta \mathrm{x}_{\min }=3.125 \mathrm{~mm}$. An overall view of the $3 \mathrm{D}$ flow and the different refinement boxes is given in figure 19 .

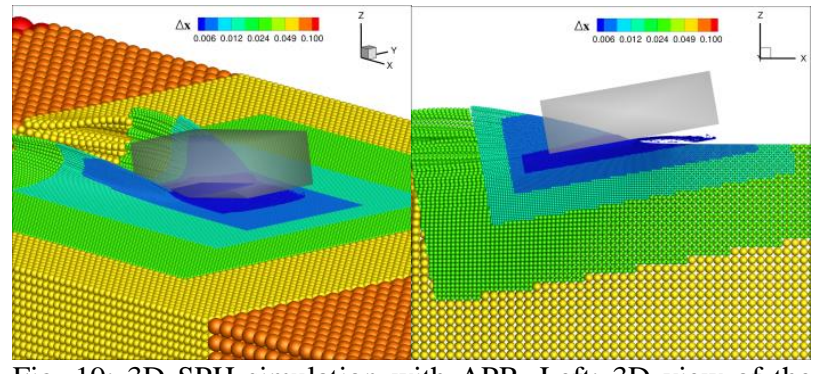

Fig. 19: 3D SPH simulation with APR. Left: 3D view of the computational domain. Right: side view at a section along the plate midline. Contours refer to the local particle size.

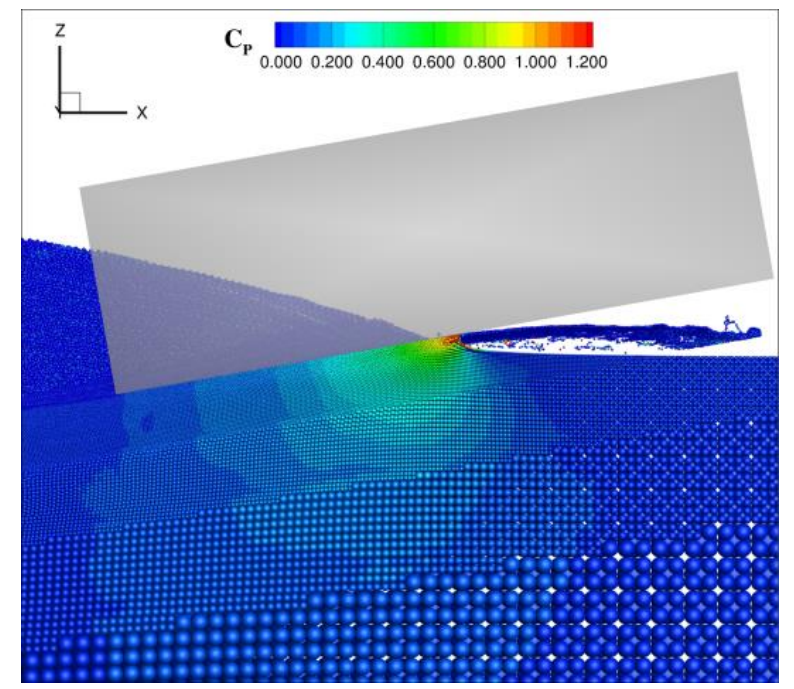

Fig. 20: side view of the pressure contour for the 3D simulation on a slice along the panel midline at $\mathrm{t}=0.03 \mathrm{~s}$.
In figure 20 the contour plot of the pressure coefficient $C_{P}$ for a particle slice at the plate midline is shown. From this plot it can be seen that the values of $\mathrm{C}_{\mathrm{P}}$ are lower with respect to those measured in the 2D simulation. This can be explained by the 3D effect associated with the flow escaping from the lateral sides of the plate as also commented in [19]. Beside this, it is worth noting how, also in the 3D case, the adopted APR technique allow for a smooth pressure field across the different refinement boxes.

The latter can be also observed in figure 21 , where a top view of the pressure field is provided. The delay of the spray root close to the lateral edges of the plate is visible in the nearly-parabolic shape of pressure peak front.

In figure 22 the comparison between 2D and 3D SPH pressure signal at P16 is provided. The 3D solution is much closer to the experimental data with respect to the $2 \mathrm{D}$ simulation. It can be clearly seen that the $3 \mathrm{D}$ effects induce a decrease of the peak amplitude and a delay of the peak occurrence, as consequence of the lower peak propagation velocity. This confirms the conjecture in [19] regarding the large discrepancies between $2 \mathrm{D}$ potential flow solution and experiments.

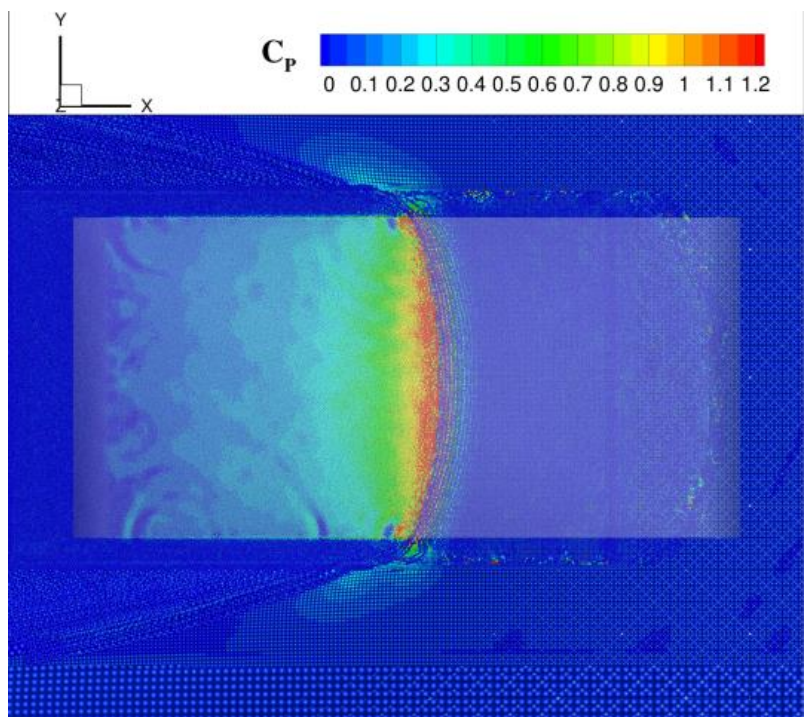

Fig. 21: top view of the pressure contour for the 3D SPH simulation at $\mathrm{t}=0.03 \mathrm{~s}$.

\section{Concluding remarks}

High speed ditching problems, in both vertical and oblique entry velocity have been discussed. All along the paper the main flow characteristics considered are the pressure signal and the jet propagation velocity (i.e. pressure peak propagation velocity) which are strictly correlated.

For the vertical water entry, the challenging problem of the impact of a flat plate with deadrise angle of $4^{\circ}$ has been considered. A critical discussion about the choice of the speed of sound as well as the key aspects to consider when comparing SPH pressure signals is 


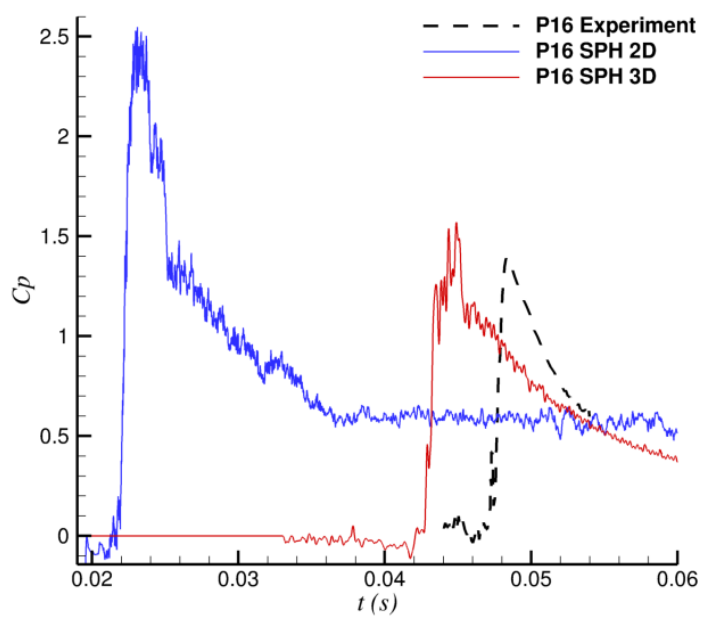

Fig. 22: comparison of the pressure signal registered at probe P16 between experiments, 2D and 3D SPH simulations.

provided. Comparisons are performed with experimental data and available analytical solutions, showing that the $2 \mathrm{D}$ weakly- compressible SPH is able to match potential flow solutions and to agree with experimental data, after careful analysis of the measure system. For this case the observed 3D effects are negligible.

Then, analysis has been focused on ditching problems of flat plates with high horizontal velocity, comparing to the experimental data and potential flow solution in [23] and [19]. For this case, an Adaptive Particle Refinement strategy has been adopted to limit the computational costs.

Test cases with pitch angle of $4^{\circ}$ and $10^{\circ}$ are considered. In both situations it is observed that, differently from the purely vertical case, the 2D SPH simulations does not correctly reproduce the experimental pressures on the plate (even if, apparently, in some under resolved cases they are quite close) even though there is still an agreement between SPH and the potential solver.

Then, a 3D simulation for the case at $10^{\circ}$ is performed recovering the agreement with the experimental data and showing that, as speculated in [19], 3D effects are substantial for this problem for both the pressure peak amplitude and the jet propagation velocity.

\section{Acknowledgements}

The research leading to these results has partially received funding from the European Union's Horizon 2020 research and innovation programme under grant agreement No 724139. The SPH simulations performed under the present research have been obtained using the SPH-Flow solver, software developed within a collaborative consortium composed of Ecole Centrale de Nantes, NextFlow Software company and CNR-INSEAN.

\section{References}

[1] H. Streckwall, O. Lindenau, and L. Bensch, "Aircraft ditching: a free surface/free motion problem," Archives of Civil and Mechanical Engineering, vol. 7, no. 3, pp. 177-190, 2007.

[2] B. Guo, P. Liu, Q. Qu, and J. Wang, "Effect of pitch angle on initial stage of a transport airplane ditching," Chinese Journal of Aeronautics, vol. 26, no. 1, pp. 17-26, 2013.

[3] G. Oger, M. Doring, B. Alessandrini, and P. Ferrant, "Two-dimensional SPH simulations of wedge water entries," J. Comp. Phys., vol. 213 (2), pp. 803 822, 2006.

[4] D. Meringolo, A. Colagrossi, S. Marrone, and F. Aristodemo, "On the filtering of acoustic components in weakly-compressible SPH simulations," Journal of Fluids and Structures, vol. to appear, 2017.

[5] S. Lind, P. Stansby, and B. D. Rogers, "Incompressible-compressible flows with a transient discontinuous interface using smoothed particle hydrodynamics (SPH)," Journal of Computational Physics, vol. 309, pp. 129-147, 2016.

[6] S. Marrone, A. Colagrossi, A. Di Mascio, and D. Le Touzé, "Prediction of energy losses in water impacts using incompressible and weakly compressible models," Journal of Fluids and Structures, vol. 54, pp. 802-822, 2015.

[7] G. Oger, S. Marrone, D. Le Touzé, and M. De Leffe, "SPH accuracy improvement through the combination of a quasi-Lagrangian shifting transport velocity and consistent ALE formalisms," Journal of Computational Physics, vol. 313, pp. 76-98, 2016.

[8] J. Vila, "On particle weighted methods and Smooth Particle Hydrodynamics," Mathematical Models \& Methods in Applied Sciences, vol. 9, no. 2, pp. 161-209, 1999.

[9] S. Marrone, A. Colagrossi, J. Park, and E. Campana, "Challenges on the numerical prediction of slamming loads on LNG tank insulation panels," Ocean Engineering, vol. 141, pp. 512-530, 2017.

[10] R. Zhao and O. M. Faltinsen, "Water entry of two-dimensional bodies," Journal of Fluid Mechanics, vol. 246, pp. 593-612, 1993.

[11] A. A. Korobkin and A. Iafrati, "Numerical study of jet flow generated by impact on weakly compressible liquid," Physics of Fluids, vol. 18, no. 3, p. 032108, 2006.

[12] E. Campana, A. Carcaterra, E. Ciappi, and A. Iafrati, "Some insights into slamming forces: compressible and incompressible phases," Proceedings of the Institution of Mechanical Engineers, Part C: Journal of Mechanical Engineering Science, vol. 214, no. 6, pp. 881-888, 2000.

[13] Korobkin, A., Pukhnachov, V. Initial stage of 
water impact. Annual Review of Fluid Mechanics 20, 159-185, 1988.

[14] O. M. Faltinsen and Y. A. Semenov, "Nonlinear problem of flat-plate entry into an incompressible liquid," Journal of fluid mechanics, vol. 611, p. 151, 2008.

[15] S. Okada and Y. Sumi, "On the water impact and elastic response of a flat plate at small impact angles," Journal of marine science and technology, vol. 5, no. 1, pp. 31-39, 2000.

[16] S.-L. Chuang, "Experiments on slamming of wedge-shaped bodies," Journal of Ship Research, vol. 11, no. 3, pp. 190-198, 1967.

[17] S. Mizoguchi and K. Tanizawa, "Impact wave loads due to slamming - a review," Ship Technology Research, vol. 43, no. 4, pp. 139-154, 1996.

[18] M. Tenzer, O. e. Moctar, and T. E. Schellin, "Experimental investigation of impact loads during water entry," Ship Technology Research, vol. 62, no. 1, pp. 47-59, 2015.

[19] A. Iafrati, "Experimental investigation of the water entry of a rectangular plate at high horizontal velocity," Journal of Fluid Mechanics, vol. 799, pp. 637-672, 2016.

[20] J. Armand and R. Cointe, "Hydrodynamic impact analysis of a cylinder," Journal of offshore mechanics and Arctic engineering, vol. 109, no. 3, pp. 237-243, 1987.

[21] T. Watanabe, "Analytical expression of hydrodynamic impact pressure by matched asymptotic expansion technique," Trans. West-Japan Soc. Naval Arch., no. 71, pp. 77-85, 1986.

[22] Y. A. Semenov and A. Iafrati, "On the nonlinear water entry problem of asymmetric wedges," Journal of Fluid Mechanics, vol. 547, pp. 231-256, 2006.

[23] A. Iafrati, S. Grizzi, M. Siemann, and L. B. Montañés, "High-speed ditching of a flat plate: Experimental data and uncertainty assessment," Journal of Fluids and Structures, vol. 55, pp. 501-525, 2015.

[24] Chiron, L., Oger, G., de Leffe, M., \& Le Touzé, D. "Analysis and improvements of Adaptive Particle Refinement (APR) through CPU time, accuracy and robustness considerations". Journal of Computational Physics, 354, 552-575, 2018. 